\title{
Research into Technology Decision Methods of CAPP Artificial Intelligence
}

\author{
Zhang Bo \\ NanChang Institute of Science \& Technology
}

\begin{abstract}
Technology decision is the core of CAPP system, and the ability of technology decision determines the main performances of the CAPP system while the way to make decisions determines the type of CAPP system. In this paper, the method of automatic processing in CAPP is studied systematically, and an effective intelligent process decision-making system is established. Some problems exist in the development of CAPP, which is useful for future research and development. In this paper, the requirement of the information model of parts in the CAD/CAPP integrated system is presented. The method to establish the information model of the parts in the process decision system is given.
\end{abstract}

Keywords-CAPP; Artificial intelligence; Database; Technology decision

\section{INTRODUCTION}

Computer-aided process planning (CAPP) is a system which is composed of human and computer. The design information is converted into a series of machining operations which are required for the manufacture of parts. It is the process of matching and optimizing all the possible processing capability of product design information and manufacturing environment. So far, CAPP has experienced 40 years. Many significant research results have been achieved. In particular, the CAPP system, neural network and fuzzy logic have been applied to CAPP. The practical effect of CAPP system has become a bottleneck for CAD/CAPP/CAM integration.

While the research of CAPP Technology (including artificial intelligence technology) is in trouble and stagnant, domestic and foreign experts and scholars put forward conception of man-machine intelligent system, the research and development of CAPP system opens up a new way to bright future full of. In 1994, Chevener Francois of the first university in France, Bernal Leon Claude, said: the problem solving often cannot be carried out automatically, because for some sub problems, the computer cannot find the corresponding solution strategy or knowledge, can only rely on people to solve, so people must be able to do the problem of automatic process. 1994-95, Lu Yongxiang, and put forward technical argument of "man-machine integration system (Humachine system), scientific system and the key technology. In 1999, at the forty-ninth CIRP annual meeting, the Swedish Qiao Bai (Kjellberg) for the theme of the report "Design of the production system for human" has been recognized by various countries, and that the twenty-first Century manufacturing system should be from the technology oriented to the people.

CAPP system in the future development direction should take the human center, the man-machine integration technology route. Research on the architecture and key technologies of the intelligent CAPP system based on knowledge, and establish a new type of CAPP system, so as to improve the application level of CAPP.

In this paper, the results are satisfied with the results of the system running and the examples. It shows that the method is reasonable and effective. The overall performance of the system will be further improved and developed in the further research and development of the system.

\section{ORIGN, DEVELOPMENT AND ClASSIFICATION OF THE CAPP TECHNOLOGY}

In the last few years, the artificial neural network technique, fuzzy reasoning and case-based reasoning are used in CAPP. It has also proposed the idea of the construction of CAPP system, and has carried out fruitful practice. There will be the traditional derivation, traditional generative method and artificial intelligence together, combining their advantages. Figure 1 shows the development of the CAPP. 


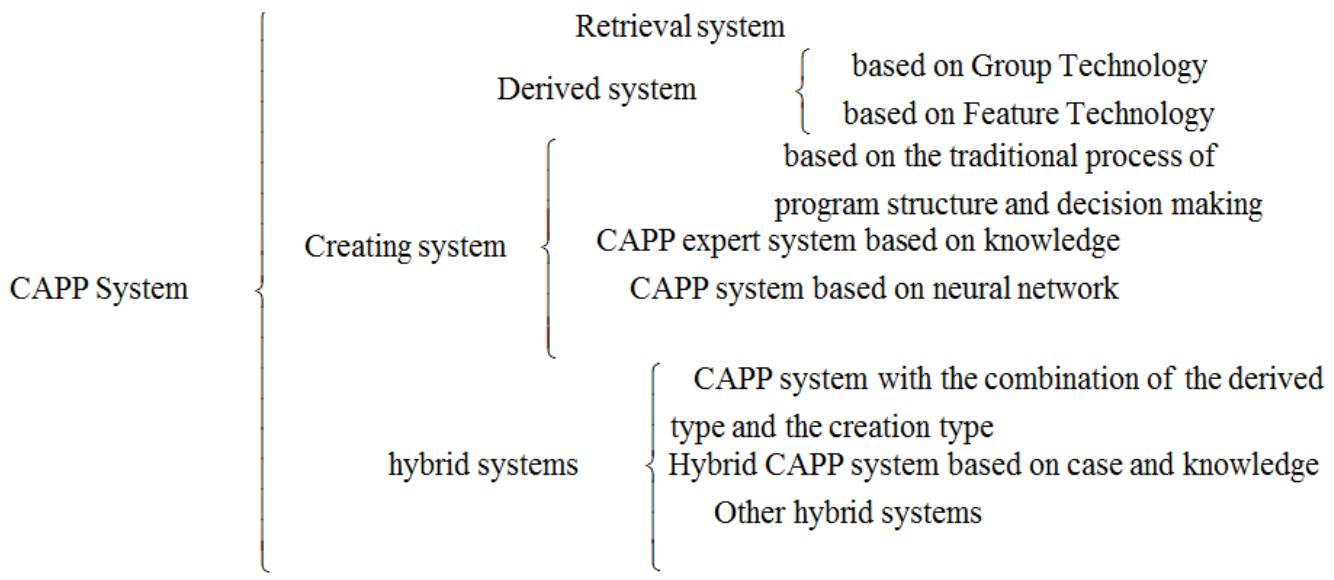

Fig 1. Classification of CAPPs Development

Analysis of CAPP development in the past 30 years and nearly 10 years in China, the development of CAPP research can be summarized as follows:

1) The development of the system of the derived system to generative.

2)From the traditional CAPP system to the intelligent CAPP system development.

3)Development of CAPP system for complex non rotating body parts by CAPP system of revolving body parts.

3)From isolated CAPP system to CAPP/NC and CAD/CAPP/CAM integration direction.

4)From the development of research and application of CAPP system to the CAPP system and the development of expert system tools, the development of CAPP system is gradually popularized and raised to a new level.

\section{SYSTEM COMPOSITION PRINCIPLES}

The method of manual design of the process for the process of the process of the process of the process of a certain way into the process of automatic or interactive design, complete with the design and process of all process documents.

System is at work. First of all, the system of man-machine interface, through Chinese menu guide, input specific parts of the manufacturing information (such as part parameters and dimensions) and process header file information (such as code, spare parts and other materials), also on the screen display to input the part surface characteristics of two-dimensional graphics. If the input is not properly, the system will give an error message, the operator can set up the function module by calling the system itself, to modify and adjust the input information. Then, the system can automatically realize the process dimension chain calculation, tolerance and margin determination by module transformation. At the end of the paper, the standard process database, such as tooling and fixture, is called to provide the original data file for the process route. Then, the system automatically turns to the process generating module, and uses the logic decision of the system, the function to judge, search or create the process of the part. Figure 2 for the system working principle diagram. 


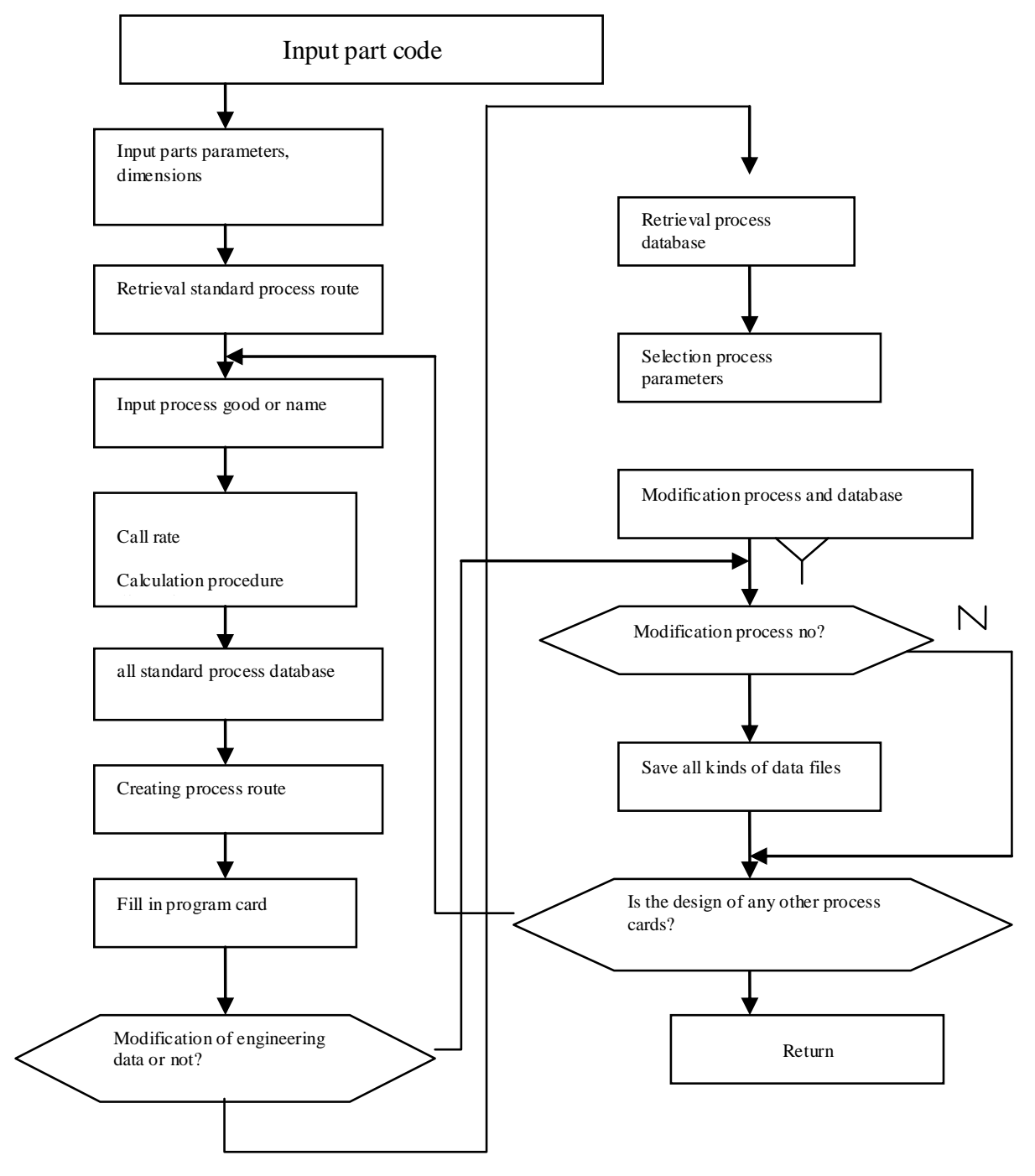

Fig 2. Diagram of the System's Work Principles

\section{TeChNOLOGY DeCISION BASED ON EXPERT}

\section{SYSTEM}

Expert system is a kind of intelligent software system, which can be used to accept and deal with the symbolic form of computer. It can solve the problem that only experts can solve in the field. Expert system is a kind of intelligent software system, which can be used to accept and deal with the symbolic form of computer. It can solve the problem that only experts can solve in the field. Because of the advantages and disadvantages of the expert system, it mainly depends on whether it has a rich knowledge of solving problems, so the expert system is also called knowledge system (Based System Knowledge).Expert system is different from the general data processing system, in the traditional process system, but simply to store the answer, people can retrieve the answer in the machine, and in the expert system is not stored in the answer, but the ability and knowledge of reasoning.

The basic structure of the expert system is composed of the knowledge base and the reasoning mechanism. Simply to say, knowledge base + reasoning $=$ expert system. Knowledge base is stored in the knowledge of a certain field obtained from the expert, and the inference engine can deduce the conclusion according to the problem. Generally speaking, different application fields and different types of expert system, the function and structure of the system are not the same. And a practical expert system, the knowledge base, database, inference engine, explanation mechanism, knowledge acquisition mechanism and user interface are six basic parts. 


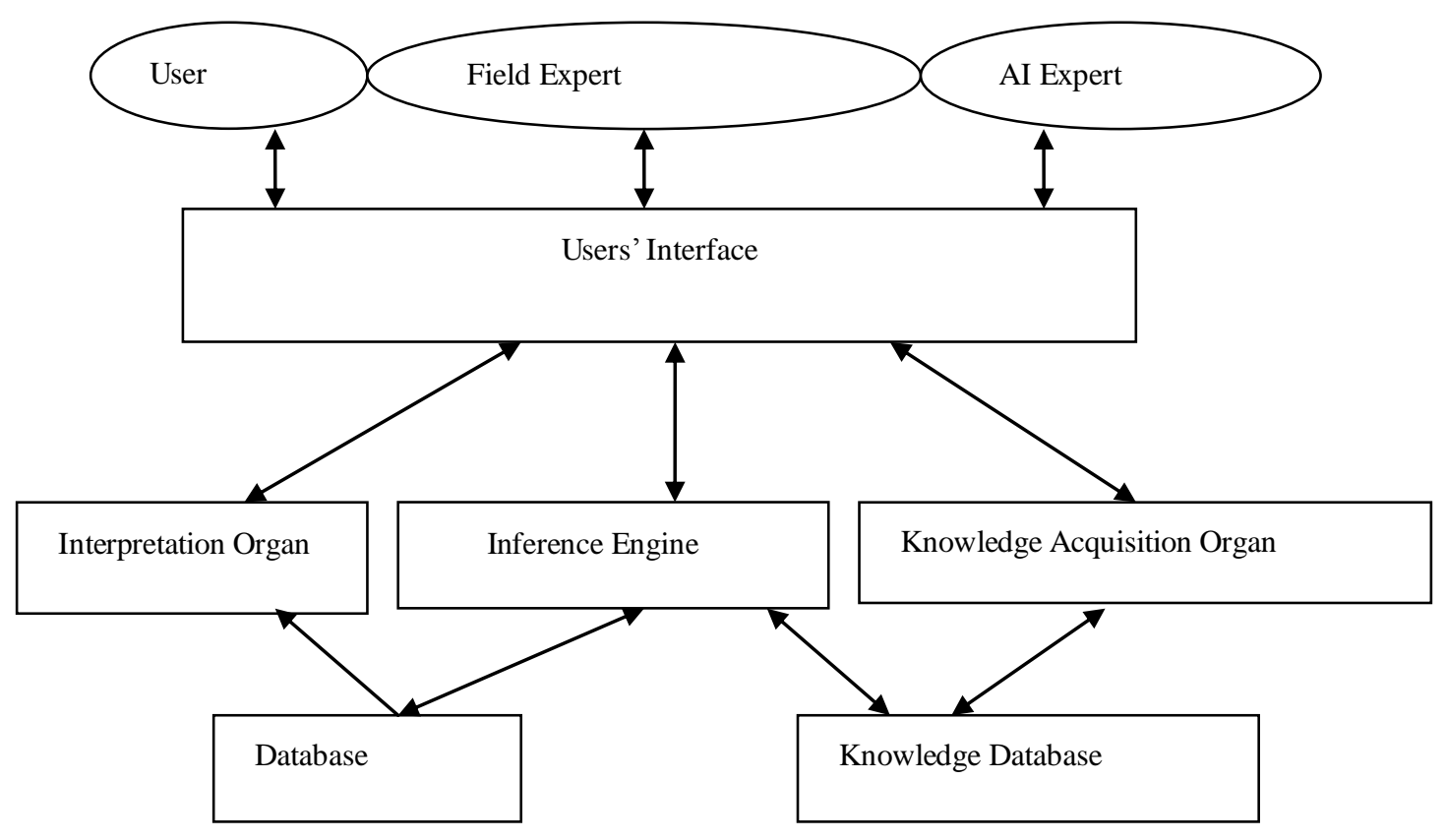

Fig 3. Basic Structure of Expert System

\section{KNOWLEDGE BASE}

Knowledge base is the knowledge storage of expert system, and it is used to store the knowledge of process rules in our process decision making expert system. In order to build the knowledge base, we need to solve the problem of how to store knowledge, this is the so-called knowledge expression, exactly, is how to use the computer to store and identify ways to express knowledge.

Database

Database also known as the global database or a comprehensive database, and is used to store the facts, data, the initial state and the goal of the process. In fact, it is equivalent to the working memory of the expert system, which is used to store the facts and conclusions of the user input. The scale and structure of the database can be determined according to the purpose of the system, and the contents of the storage are determined according to the needs of the system. For example, in our process decision-making expert system, the database of the parts of the input information, decision-making results and so on.

\section{CONCLUSION}

In this paper, the automatic process planning and key technologies of CAPP are studied, and the system of intelligent process decision system based on expert system is developed with the application of the system. The acquisition, scientific management and rational use of information are the important parts of developing CAPP system. It directly affects the degree of automation of process generation. This paper aims to explore a way to use the existing advanced theory and software tools to enhance the practicability and automation of CAPP system.

\section{REFERENCES}

[1] Elmaraghy H A. Evolution and Future Perspectives of CAPP[J]. CIRP Annals - Manufacturing Technology, 1993 , 42(07):739-751.

[2] Fishbein J D, Dobrowsky $\mathrm{R} T$, Bielawska $\mathrm{A}$, et al. Ceramide-mediated growth inhibition and CAPP are conserved in Saccharomyces cerevisiae.[J]. Journal of Biological Chemistry, 1993, 268(13):9255-9261.

[3] Behren R V, Condit J, Zhou F, et al. Capriccio: Scalable Threads for Internet Services[J]. Proceedings of Acm Symposium on Operating Systems Principles, 2003, 37(5):268--281.

[4] Gandolfo M A, Crepet K C N L. A new fossil flower from the Turonian of New Jersey: Dressiantha bicarpellata gen. et sp. nov. (Capparales).[J]. American Journal of Botany, 1998, 85(7):964-974.

[5] Jung-Youn L, Byung-Chun Y, Rojas M R, et al. Selective trafficking of non-cell-autonomous proteins mediated by NtNCAPP1.[J]. Science, 2003, 299(5605):392-396.

[6] X. W. Xu *, H. Wang, J. Mao, et al. STEP-compliant NC research: the search for intelligent CAD/CAPP/CAM/CNC integration[J]. International Journal of Production Research, 2005, 43(17):3703-3743.

[7] Rodman J E, Sytsma K J. Molecules, morphology, and Dahlgren's expanded order Capparales.[J]. Systematic Botany, 1996, (3):289-307.

[8] Kruth J P, Detand J. A CAPP system for non-linear process plans[J]. CIRP Annals-Manufacturing Technology, 1992, 41(07):489-492.

[9] Naeye R L. Thrombotic state after a hemorrhagic diathesis, a possible complication of therapy with epsilon-aminocapproic acid.[J]. Blood, 1962, 19(6):694-701.

[10] Mu Z, Xiao H. Computer Aided Process Planning System Design Based on Semi-Generative[J]. International Review on Computers \& Software, 2012. 